\title{
Quaderni
}

QUADERNI Communication, technologies, pouvoir

71 | Hiver 2009-2010

Le changement climatique : les résistances à

l'adaptation

\section{Le changement climatique : conséquences pour les végétaux}

Bernard Seguin

\section{(2) OpenEdition}

Journals

Édition électronique

URL : http://journals.openedition.org/quaderni/525

DOI : 10.4000 /quaderni.525

ISSN : 2105-2956

Éditeur

Les éditions de la Maison des sciences de l'Homme

Édition imprimée

Date de publication : 5 janvier 2010

Pagination : 27-40

Référence électronique

Bernard Seguin, "Le changement climatique : conséquences pour les végétaux », Quaderni [En ligne], 71 | Hiver 2009-2010, mis en ligne le 05 janvier 2012, consulté le 30 avril 2019. URL : http:// journals.openedition.org/quaderni/525 ; DOI : 10.4000/quaderni.525 


\section{$D$ ossier}

\section{le changement}

climatique : conséquences

\author{
pour les \\ végétaux
}

Bernard

Seguin

Directeur de recherches à l'INRA

Mission "Changement

climatique et effet de serre"
Le changement climatique n'est, bien sûr, qu'un des déterminants qui vont conditionner l'évolution de l'agriculture au cours du $\mathrm{XXI}^{\mathrm{e}}$ siècle. La remise en question de sa vocation unique de production d'alimentation, déjà largement entamée depuis quelques années par la reconnaissance de sa multifonctionnalité, est fortement accentuée actuellement par l'ouverture des perspectives de l'utilisation de la biomasse pour la substitution d'énergie fossile (biocarburants, cultures énergétiques).

Le toutse situe dans un contexte de transformation profonde du contexte économique, incluant les réformes de la PAC (Politique Agricole Commune).

Cependant, l'agriculture reste sans aucun doute, parmi les activités humaines, une de celles le plus directement influencées par le climat, malgré l'augmentation de sa productivité dans le cas des pays développés.

Le changement climatique aura donc un impact sur la composante biotechnique de la production.

Accroissement de la teneur en gaz carbonique et autres gaz à effet de serre dans l'atmosphère, élévation de la température, modification des régimes pluviométriques, et donc des différents termes du bilan hydrique (évaporation, drainage, ruissellement), évolution de la couverture nuageuse, et donc du bilan radiatif : l'ensemble des facteurs bioclimatiques qui régissent le fonctionnement des écosystèmes est amené à se modifier, et il faut donc en premier lieu prévoir et quantifier ces modifications et leurs conséquences. 


\section{Les impacts futurs sur les végétaux}

\section{Conséquences pour la production végétale}

Legazcarbonique favorise la croissance végétale. Les plantes fixent le $\mathrm{CO}_{2}$ de l'atmosphère grâce à une molécule organique dont le nombre d'atomes de carbone diffère selon les espèces : les plantes en $\mathrm{C} 3$ (3 atomes de carbone) et celles en $\mathrm{C} 4$ (4 atomes de carbone) réagiront différemment à une variation de la quantité du $\mathrm{CO}_{2}$ atmosphérique. Si cette dernière double d'ici à la fin de ce siècle, la photosynthèse brute augmentera de $30 \%$ pour les plantes en C3 (blé et riz)etde $15 \%$ pourcellesenC $4 \mathrm{~d}$ 'originetropicale, comme le maïs. Les premières assimileront $20 \%$ de carbone en plus, les secondes $10 \%$. La production de biomasse devrait alors augmenter. Cet effet sur la photosynthèse se combinant aux variations de températures et de précipitations, les conséquences sur le rendement des espèces cultivées ou la productivité forestière seront cependant plus contrastées qu'une simple augmentation de la production de biomasse. Les plantes possèdent en effet une température optimale pour la photosynthèse, qui est souvent déjà atteinte, voire dépassée dans le Sud de la France. Par ailleurs, dans les conditions tempérées, l'élévation de température favorise la plupart des processus physiologiques (avec, là aussi, des seuils tels que, par exemple, $36^{\circ} \mathrm{C}$ pour la viabilité du pollen de maïs). Mais elle accélère aussi le rythme de développement des cultures annuelles, de sorte qu'elle raccourcit leurs cycles et, par suite, la durée de fonctionnement de la photosynthèse. À l'inverse, pour les plantes à cycle non déterminé (en particulier les forêts), la saison de croissance, qui commencera plus tôt au printemps et finira plus tard à l'automne, sera allongée. Au final, le bilan de la production de biomasse dépendra à la fois du type de couvert, des conditions climatiques et des pratiques de culture ou de conduite des couverts végétaux.

Il existe encore assez peu d'études spécifiques sur les conséquences pour les végétaux d'ornement et les couverts urbains. Ce sont donc celles, beaucoup plus documentées, sur les espèces cultivées (en agriculture) ou gérées (en sylviculture) qui peuvent nous donner les indications les plus complètes pour avoir une idée des grandes lignes des impacts attendus.

\section{Les impacts attendus en agriculture}

En prenant le cas de la France, au niveau des grandes cultures, les résultats des simulations effectuées à l'INRA avec les modèles de culture sur le blé et le maïs (Delecolle et al 1999) permettent de conclure à des effets légèrement positifs sur le premier, avec des augmentations de rendement allant de $2,5 \%$ à $5,7 \%$, et des effets plus variables sur le maïs $(+10 \%$ à - $16 \%$ dans le cas d'une culture irriguée dans le Sud-Est). Ils sont en bon accord avec les études comparables d'autres pays pour les conséquences sur les céréales en milieu tempéré (figure 1, d'après Easterling et al 2008). Au niveau des prairies (Soussana 2001), la conjugaison de travaux expérimentaux (sous serre et en enrichissement naturel à l'extérieur) et de modélisation à partir d'un modèle d'écosystème prairial conduit à envisager, dans les conditions du Massif Central, une augmentation de la production de biomasse aérienne de l'ordre de $25 \%$ (dont $18 \%$ attribuables au seul doublement de $\mathrm{CO}_{2}$ ). 
Figure 1. Effet du réchauffement sur le rendement du blé et du maïs en zone tempérée avec indication des effets possibles de l'adaptation (d'après Easterling et al., 2008).
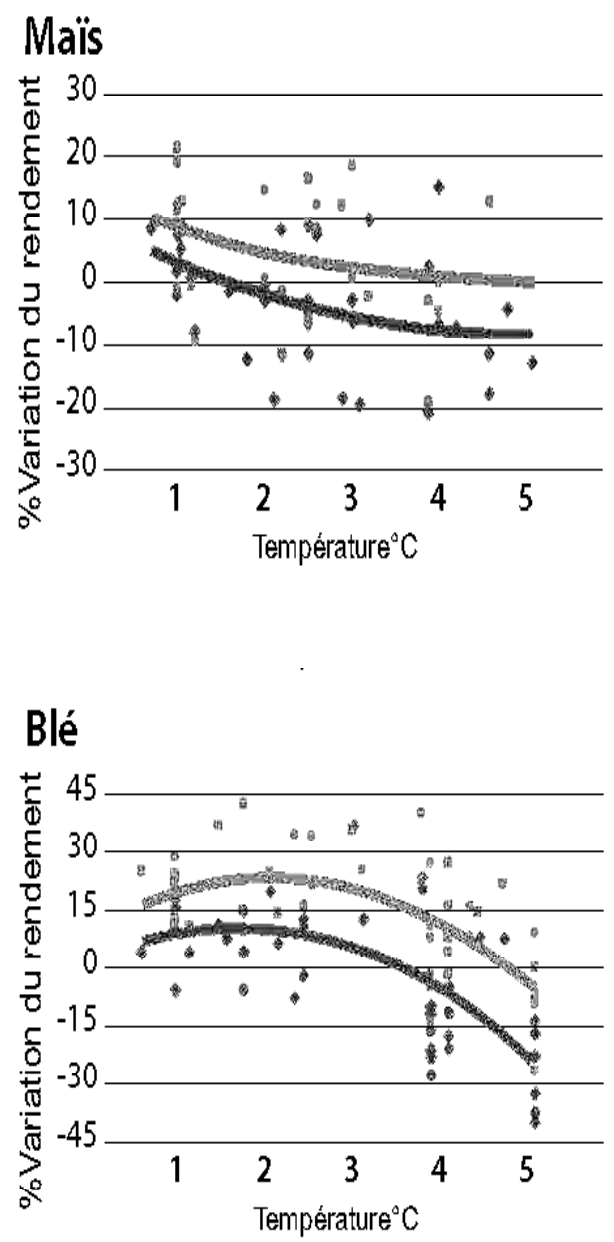

sans adaptation avec adaptation (d'après Easterlinget al.2007)
En termes de système d'élevage, la valorisation de cette augmentation de production devrait permettre une augmentation du chargement animal (en gros de 20\%) ou une augmentation de la saison de pâturage de l'ordre de trois semaines, avec un accroissement de l'ingestion de 7 à $20 \%$ et de 2 à $20 \%$ pour la production de viande. Mais ce potentiel plus fort pourrait être très largement contrebalancé par l'effet négatif du stress hydrique, en particulier dans les régions du Sud de la France, mais la perspective de sécheresses plus fortes amène aussi à envisager des effets significatifs de la sécheresse dans l'Ouest et même le Nord de la France.

Pour les cultures pérennes, essentiellement arbres fruitiers et vigne (Seguin et al 2004), le facteur primordial devrait être l'avancée des stades phénologiques, d'autant plus marquée que l'on s'éloigne en cours de saison de la levée de dormance, qui risque elle d'être plus tardive à cause du manque de froid. Pour certaines espèces telles que l'abricotier, les hivers doux risquent même de créer des troubles physiologiques (chute de bourgeons, fruits avortés). Ensuite, l'action de la chaleur reprendra vite le dessus et l'avancée de la date de floraison peut conduire paradoxalement à augmenter le risque de gel (figure 2), et à envisager des conditions climatiques moins favorables pour la fécondation et la pollinisation, en dépit du réchauffement des températures. Pour la vigne, la période de la maturation sera décalée (pour le Midi, avancée d'après le 15 août à courant juillet selon Lebon 2002), ce qui a pour effet de 'démultiplier' le réchauffement, en ajoutant au seul effet climatique un effet lié à l'avancée du calendrier, avec des conséquences certaines sur 
la qualité de la vendange, plus chargée en sucre (et donc en degré alcoolique) et moins en acide.

Il faudra aussi considérer l'impact sur les adventices (mauvaises herbes) et les insectes et maladies cryptogamiques, encore mal cerné à l'heure actuelle.

Les tendances que nous venons de détailler dans le cas de la France se retrouvent dans leurs grandes lignes au niveau européen (Olesen et Bindi 2002), avec la perspective de conditions finalement globalement plus favorables pour le Nord du continent, et à l'inverse avec des impacts plutôt négatifs dans le Sud, la ligne de partage se retrouvant au niveau français. C'est évidemment une vision simplifiée, très schématique, qui est actuellement de plus en plus complétée dans le détail des régions et des cultures par un grand nombre d'études. Il n'en reste pas moins que ce partage se retrouve en tendance dans les projections au niveau du globe (Easterling et Apps 2005, Easterling et al. 2008), avec des productions fortement pénalisées dans les climats des pays à faible latitude, alors que celles des pays de climat tempéré pourraient être légèrement favorisées, du moins tant que le réchauffement reste inférieur à 2 ou $3^{\circ} \mathrm{C}$.

Figure 2. Effet du réchauffement climatique sur les dégâts de gel simulés pour 3 productions fruitières (pommier, abricotier, pêcher) sur le site d'Avignon.

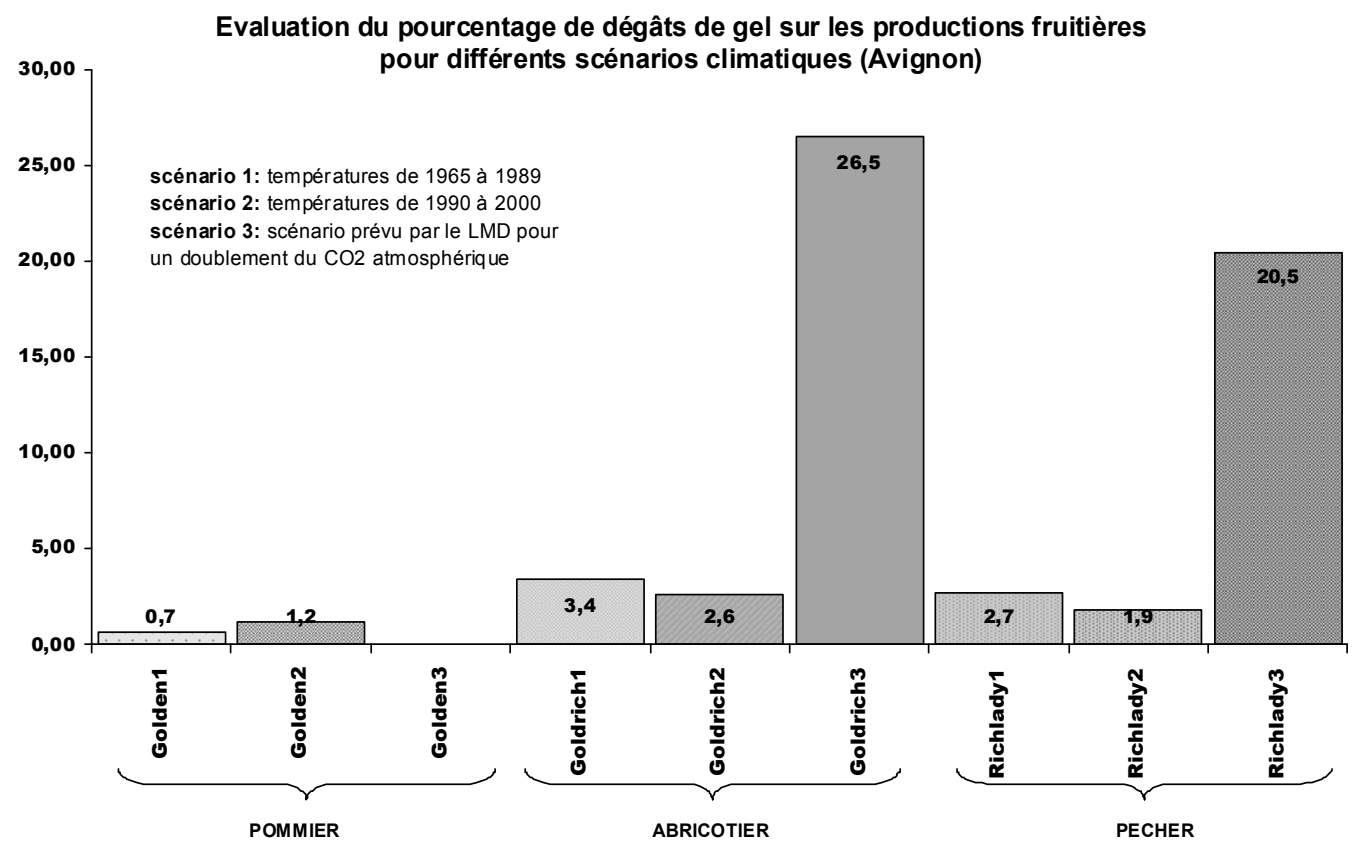


Mais au-delà, le réchauffement devrait aussi se traduire par une chute des rendements, même limitée par rapport aux impacts dans le Sud.

Il faut relativiser la portée de ces projections en notant qu'il faudrait aussi considérer l'impact sur les adventices (mauvaises herbes) et les insectes et maladies cryptogamiques, encore mal cerné à l'heure actuelle.

\section{Quels impacts pour la forêt?}

Pour les forêts, le schéma général est assez semblable : stimulation de la photosynthèse par l'accroissement du $\mathrm{CO}_{2}$ atmosphérique, allongement de la saison de croissance et températures plus favorables vont donner, dans un premier temps, des conditions de croissance plus favorables pour les forêts tempérées, à l'inverse des forêts tropicales. Par contre, pour des valeurs de réchauffement plusélevées (de l'ordre de 2 à $3^{\circ}$ C), le mouvement va s'inverser et la productivité des forêts tempérées diminuera, alors que celle des milieux tropicaux sera fortement réduite par l'effet combiné des fortes températures et de la sécheresse accentuée. Ce schéma général est bien illustré par les projections sur la forêt française effectuées dans le cadre du projet CARBOFOR soutenu par le projet GICC (Loustau et al. 2004): les modèles de fonctionnement des couverts forestiers montrent que la stimulation de la croissance des arbres par la seule réponse de la photosynthèse brute à l'augmentation du gaz carbonique devrait atteindre environ $40 \%$, pour l'ensemble de la phase juvénile. Ce chiffre est plus élevé pour les feuillus que pour les résineux. Lorsque, à l'aide des modèles, on combine ces effets à ceux des modifications climatiques, on obtient, comme pour les cultures, des résultats contrastés suivant les espèces et les régions. Le débourrement serait plus précoce de 6 à 10 jours pour les feuillus, et de 15 à 20 jours en moyenne pour le pin maritime. Pour le pin sylvestre et l'épicéa, qui ont des besoins en froid plus élevés, l'avancée du débourrement ne serait perceptible qu'en altitude; en plaine il y aurait un retard. Pour toutes les espèces, le risque de gel tardif serait diminué. Au total, si la production nette en France devrait bien augmenter, l'augmentation se situerait nettement en dessous des chiffres donnés par le seul effet de stimulation de la photosynthèse (de 2 à $15 \%$ ), avec surtout une forte variabilité suivant les localisations en fonction des conditions hydriques et thermiques locales et de la fertilité des sols. Les effets prédits sont globalement positifs dans le nord de la France pour les feuillus sociaux avec une réponse décroissante de l'Est vers l'Ouest, la façade nord-ouest présentant même une anomalie de production nette négative. Dans le Sud-Ouest ils sont positifs en début de siècle puis s'inversent avec une anomalie négative augmentant vers l'intérieur des terres en fin de siècle. En zone méditerranéenne, l'accentuation de sécheresse estivale conduirait aux mêmes effets négatifs, renforcés par l'augmentation des risques d'incendie. Outre l'aggravation du risque météorologique dans les régions affectées traditionnellement, il faut s'attendre à une extension géographique vers des régions qui n'y sont, ni préparées, ni habituées (Rigolot 2008).

\section{Les moyennes et les extrêmes}

Les éléments qui viennent d'être présentés s'appuient uniquement sur les valeurs moyennes 
des facteurs climatiques.

L'éventualité d'évènements extrêmes et, de façon plus large, la prise en compte de la variabilité de ces facteurs pourraient conduire à des impacts différents de ce réchauffement moyen continu par le dépassement de valeursseuils encore mal cernées. C'est assez évident pour les sécheresses (comme l'ont récemment montré 2003, puis 2005 et 2006) ou les fortes pluies (qui affectent l'agriculture par l'érosion et l'inondation des parcelles), mais c'est également vrai pour la température. D'abord par ses valeurs basses pour les gels d'hiver ou de printemps (évoqué ci-dessus): si les scénarios s'accordent pour prévoir des hivers plus doux en moyenne, l'éventualité d'épisodes de froid dévastateurs (tels qu'en 1956 ou en 1987) pour les oliviers, les agrumes ou le mimosa n'est pas à écarter, alors que les seules températures moyennes leur permettraient de remonter vers le Nord le long de la vallée du Rhône, par exemple. Ensuite par les températures élevées: la fréquence des canicules, avec des températures dépassant les $35^{\circ} \mathrm{C}$, est prévue comme devant atteindre une année sur deux vers 2050, alors que la tolérance des écosystèmes actuels, qu'ils soient cultivés ou naturels, est bien mal connue.

Enfin, pour les forêts, et comme cela a été clairement démontré par les épisodes de 1999, puis 2008, les tempêtes sont à coup sûr un élément majeur à prendre en compte, tant elles sont capables de mettre à bas en quelques instants une part significative de la production forestière accumulée sur plusieurs années. À ce niveau, et un peu comme pour les ouragans, le débat est encore ouvert chez les spécialistes sur leur renforcement dans le cadre du changement climatique ; mais il faut de toute évidence adapter la sylviculture, comme on doit l'adapter aux situations de sécheresse persistante, comme cela a été le cas dans le Sud-Est de 2003 à 2008 (voir le numéro spécial de Forêtméditerranéenne 2008).

\section{Des effets deja observables}

Les données climatiques mettent maintenant en évidence un réchauffement significatif au cours du $\mathrm{XX}^{\mathrm{e}}$ siècle sur le territoire français (Moisselin et al.2002), en accord avec ce qui est observé au niveau global (GIEC 2007a). On peut en trouver des confirmations significatives dans les observations sur les couverts végétaux, également à l'échelle du globe (GIEC 2007 b), mais particulièrement dans l'hémisphère nord et sur le continent européen, comme dans le cas de la France.

Des perturbations dans le calendrier du développement des arbres fruitiers ont été observées à la suite du réchauffement récent. La levée de dormance tend à être plus tardive par manque de froid. Pour certaines espèces, telles que l'abricotier, les hivers doux peuvent même créer des troubles physiologiques (chute de bourgeons, fruits avortés). Puis, sous l'action de la chaleur, les arbres fleurissent plus tôt. La floraison des pommiers s'est avancée d'une dizaine de jours en 30 ans (figure 3). Paradoxalement, les arbres sont plus vulnérables au gel et en dépit du réchauffement des températures, la fraîcheur du climat à une période plus précoce est moins favorable à la fécondation et à la pollinisation. 
Figure 3. Évolution de la période de floraison de la poire Williams depuis 1962.

\section{Évolution de la période de floraison (F2) de la poire Williams depuis 1962}

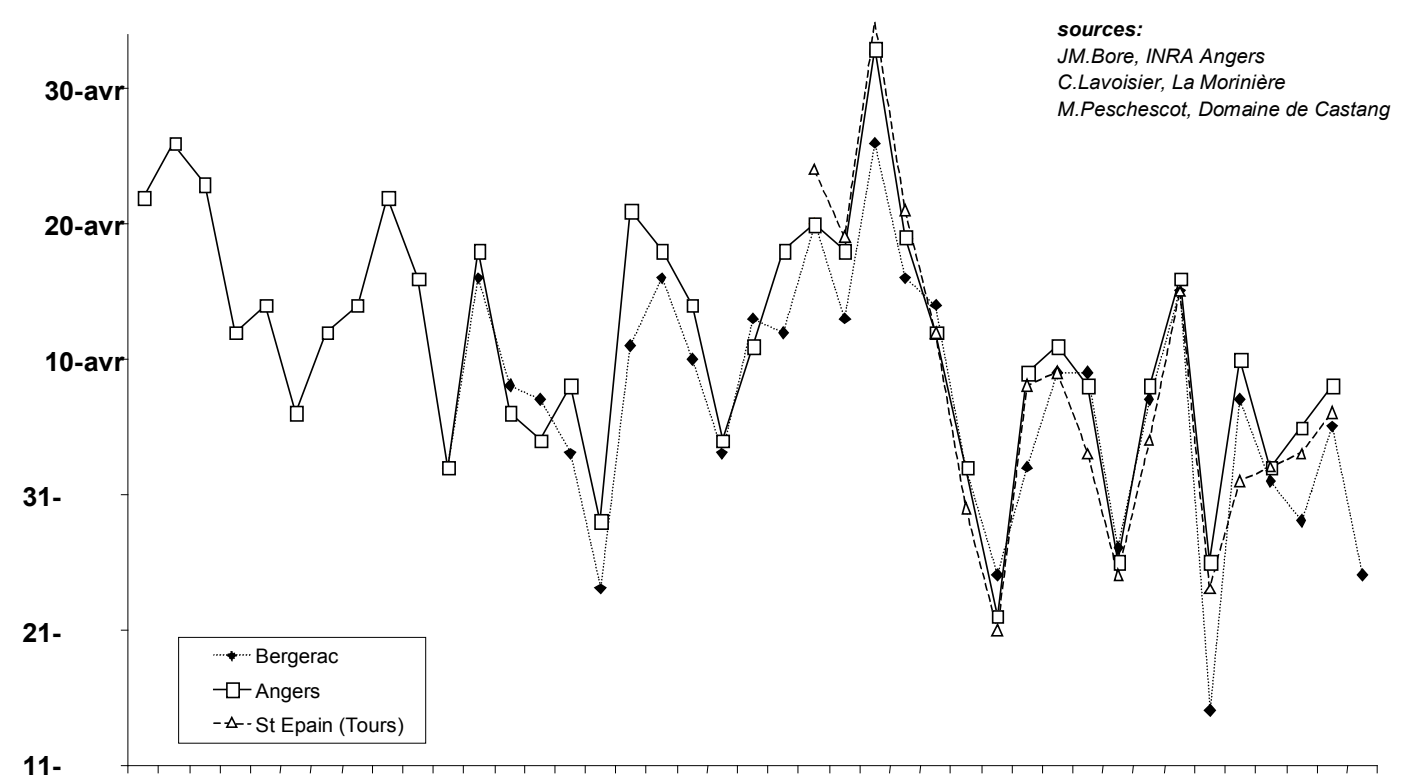

196219641966196819701972197419761978198019821984198619881990199219941996199820002002

La vigne est également plus précoce dans son calendrier. Dans toutes les régions viticoles, l'avancée moyenne de la vendange au cours des trente dernières années est de deux à trois semaines (un mois au cours des cinquante dernières années dans le Sud-Est, voir figure 4). L'augmentation de température moyenne s'est traduite par des conditions globalement plus favorables, avec moins de variabilité interannuelle pour tous les vignobles français, qui ont permis une augmentation de la teneur en alcool (de 1 à 2 degrés) et une diminution de l'acidité (voir Duchêne et Schneider 2004 pour l'Alsace), suffisamment accentuée ces dernières années pour commencer à poser des problèmes aux viticulteurs.

La même avancée phénologique est également détectable pour les forêts, qui ont aussi notablement augmenté leur productivité depuis 1960 (de l'ordre de 30 à $40 \%$ ), sans qu'il soit encore possible de séparer les effets de l'augmentation du gaz carbonique, du réchauffement ou des dépôts atmosphériques d'azote dans cette augmentation. Il faut aussi noter les premières observations de modification de la fréquence de certaines espèces dans les forêts françaises (Cluzeau et al. 2001) et 
européennes (Klötzli et Walther 1999) qui pourraient être reliées au réchauffement climatique récent. De façon générale, on constate que les espèces à feuilles persistantes et larges ont eu tendance à progresser au cours des dernières années. Le houx par exemple a vu sa surface d'extension doubler dans les Ardennes entre les années 1980 et 1990, d'après les données de l'Inventaire forestier national. Quelques observations commencent à être publiées dans la littérature internationale à propos de migrations géographiques : ainsi pour l'étude de Lenoir et al (2008) qui ont détecté une tendance vers le haut de l'ordre de $29 \mathrm{~m}$ par décade pour l'altitude optimale de 171 plantes forestières dans les montagnes de l'ouest de l'Europe. Comme l'indiquent Dupouey et Robin (2007), il s'agit pour le moment de cas emblématiques, mais pas encore d'un processus massif.

Au niveau des insectes et des maladies, il existe des observations de changements dans les populations observées, ainsi que d'apparition de nouvelles maladies ou de nouveaux ravageurs, généralement caractéristiques de climats plus chauds. Mais il est encore difficile de les attribuer spécifiquement au réchauffement climatique, tellement les nombreux autres déterminants peuvent jouer un rôle décisif. Par contre, le réchauffement récent a joué un rôle certain dans l'extension, bien documentée, vers le Nord et en altitude de la chenille processionnaire du pin (Roques et Negeilsen 2007). Il a pu également être observé une évolution sur le cycle du carpocapse des pommes qui a vu l'apparition d'une troisième génération et une augmentation de la diversité des populations de pucerons, accompagnée d'une précocité accrue des périodes d'activité. À l'inverse, on a pu noter une extinction (temporaire) du phoma du tournesol dans le Sud-Ouest, fortement défavorisé par l'augmentation des températures supérieures à $32^{\circ} \mathrm{C}$ et éradiqué après la canicule de 2003 .

\section{Il va bien falloir s'adapter}

La confrontation des projections avec les observations récentes, que ce soit directement

Figure 4. Évolution des dates de vendange à Châteauneuf-du-pape (Ganichot 2002).

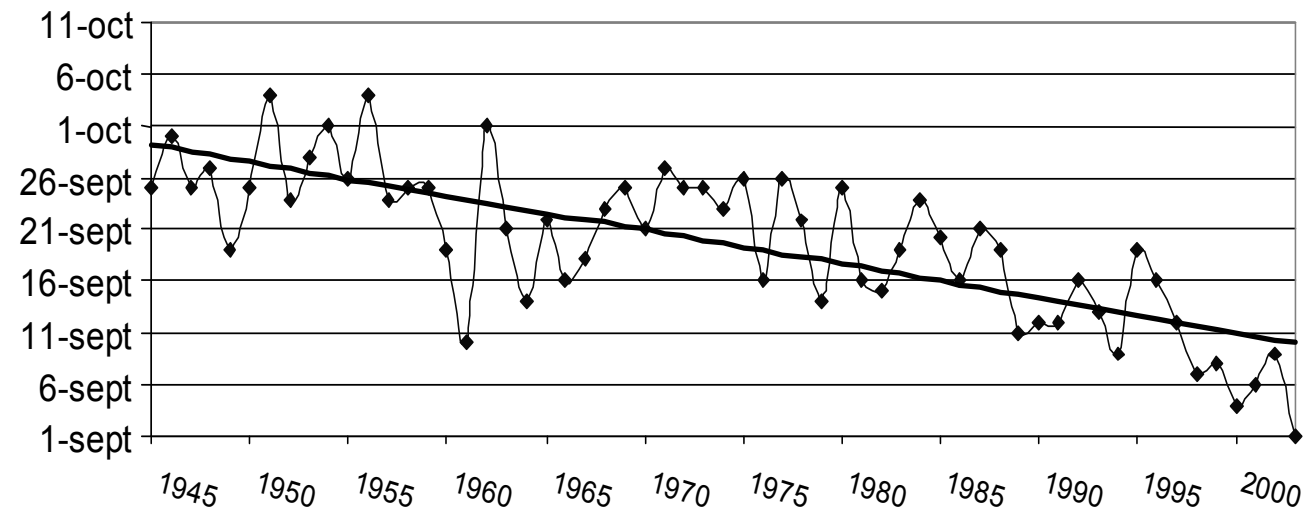


au niveau des variables climatiques (GIEC 2007a) ou plus largement à celui des impacts (GIEC 2007b) attestent d'un réchauffement significatif depuis 1860 au niveau global, avec une accentuation marquée depuis les années 1980 (voir Moisselin et al 2002 pour la France). Il est évidemment variable à la fois dans le temps et dans l'espace, mais la tendance générale parait indiscutable et bien établie par les impacts directs ou indirects sur les milieux naturels et certaines activités humaines.

Les observations prennent en compte essentiellement les effets moyens du réchauffement observé depuis une vingtaine d'années, avec, pour les conséquences sur la phénologie (Seguin et al 2004) une rupture diagnostiquée à la période 1985-1989. En complément de cette tendance de fond, les années récentes ont vu se manifester des évènements climatiques que l'on peut qualifier d'exceptionnels (par leur écart avec les valeurs normales ou moyennes). Manifestement, ces épisodes ont des impacts instantanés, ou plus tardifs compte-tenu des répercussions ultérieures, d'unordre degrandeurégalounotablement supérieur à celui de l'évolution moyenne. Il est certain que celle-ci sera déterminante à long terme, mais que la variabilité et son évolution en termes d'évènements extrêmes jouera un rôle tout aussi important dans le futur. C'est, sans doute, à l'heure actuelle, le facteur limitant pour prétendre pronostiquer ou préfigurer l'impact du changement climatique sur les écosystèmes en général, et cultivés en particulier.

Tout en étant conditionnées par le poids de l'économie, l'agriculture et la sylviculture devront s'adapterà ces changements climatiques. Dans tous les cas de figure, la disponibilité de l'eau sera le facteur limitant majeur, que ce soit l'eau stockée dans les sols ou celle nécessaire pour l'irrigation. Les stratégies de réponse de l'agriculture à la sécheresse (voir Amigues et al 2006 pour la synthèse de l'expertise collective INRA 2006) seront déterminantes. La capacité d'adaptation des cultures pérennes paraît moins rapide (10 à 20 ans). D'ores et déjà se pose la question d'éviter des variétés d'arbres fruitiers trop sensibles aux hivers doux ou au gel. Par son lien au terroir (il n'est pas envisageable de délocaliser les AOC), la vigne pose des problèmes spécifiques. Elle a montré en 2003 une bonne capacité d'adaptation à ces conditions extrêmes. La perspective de connaître régulièrement de telles conditions interroge sur la capacité de conserver à long terme la typicité ancestrale : cela paraît possible avec un réchauffement modéré, plus problématique dans une hypothèse de 4 à $5^{\circ} \mathrm{C}$.

Une remontée des cultures est envisageable, à l'instar de ce qui pourrait se passer pour les espèces forestières, dont les cartes de potentialité bioclimatique seront profondément bouleversées (Badeau et al. 2007) : un réchauffement de $1^{\circ} \mathrm{C}$ équivaut à environ $180 \mathrm{~km}$ vers le Nord. En zones de montagne, où $1^{\circ} \mathrm{C}$ correspond à $150 \mathrm{~m}$ en altitude, de nouvelles potentialités pourraient apparaître. Le réchauffement pourrait aussi permettre l'extension de cultures traditionnellement réservées au Sud (maïs-grain, sorgho, tournesol, etc.), pour lesquelles le Nord deviendrait concurrentiel, avec une alimentation hydrique plus assurée? Quid pour le Sud ? Il ne serait sans doute pas impossible techniquement 
d'introduire des cultures de pays chauds ou, par exemple, d'étendre la culture de l'olivier au Sud-Ouest, mais pour quels marchés?

Quelle que soit l'action collective sur les émissions de GES, il apparait maintenant très peu probable d'échapper à un réchauffement global d'au minimum 2 à $3^{\circ} \mathrm{C}$ pour la fin $\mathrm{du}$ siècle, et en conséquence inévitable de prévoir des mesures d'adaptation (Howden et al., 2007), variables suivant les productions et les régions, mais qui auront un socle commun. $\mathrm{Au}$ niveau français, des actions sont en cours du côté institutionnel (en particulier le ministère de l'Agriculture et de la Pêche) pour approfondir l'insertion de l'agriculture dans le schéma stratégique d'adaptation au changement climatique, dont le MEEDDM assure la responsbilité par le biais de l'ONERC. Elles peuvent s'appuyer sur une écoute attentive des milieux professionnels, qu'ils soient agricoles ou sylvicoles, directement branchés sur les réalités de terrain et à ce titre beaucoup plus sensibles à la réalité du changement climatique que d'autres catégories socio-professionnelles. La canicule/ sécheresse de 2003, puis les épisodes d'été chauds et secs qui ont suivi en 2005 et 2006 ont fortement sensibilisé les acteurs de terrain. En premier lieu les forestiers, dont les options actuelles vont produire leurs effets dans 50 à 100 ans, ce qui a pour effet de mobiliser l'ensemble de la filière sur les décisions de gestion (Legay et al.2007). Dans le cadre de l'agriculture, nous avons vu que la vigne se situait au premier rang des productions affectées, et là aussi la filière, depuis l'IFV jusqu'aux caves coopératives et aux producteurs individuels est particulièrement attentive à l'évolution et demandeuse d'options pour le futur. L'arboriculture doit aussi anticiper, compte-tenu de son temps de réponse d'une

Figure 5. Répartition géographique de 7 groupes ( $A$ à gauche) en fonction du climat actuel et ( $B$ à droite) projetée en 2100 (Badeau et al. 2007).
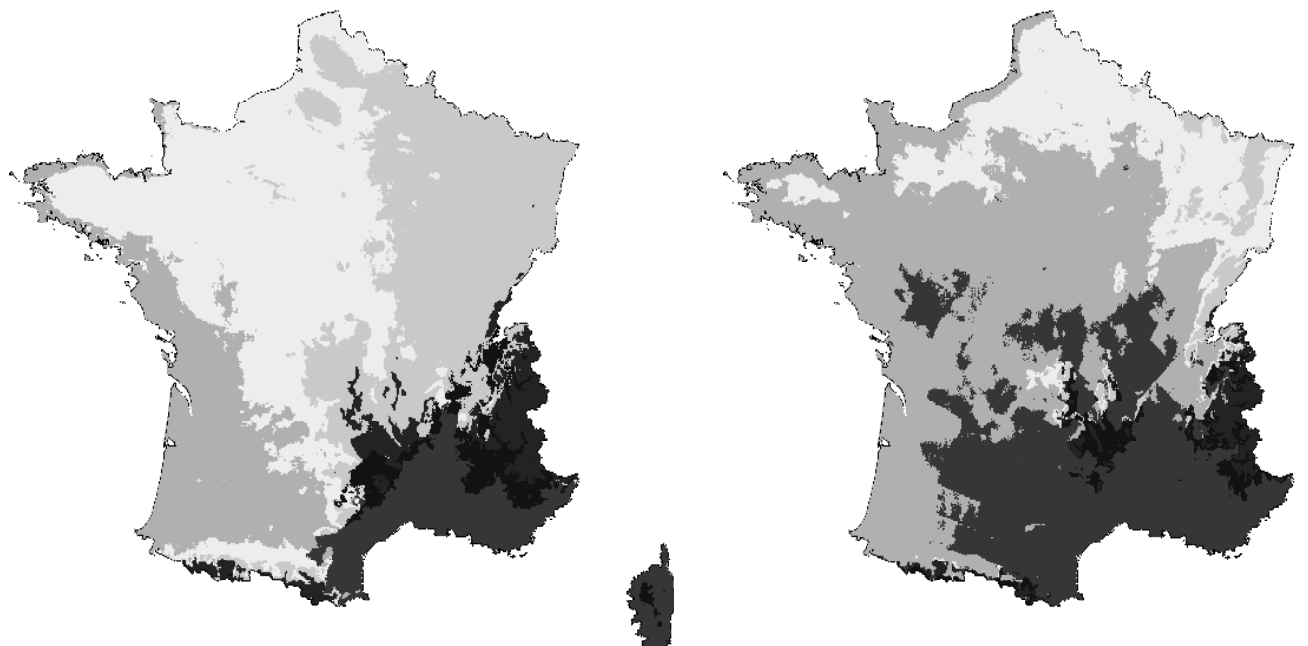
vingtaine d'années, mais les questionnements se focalisent sur la question du gel, ou éventuellement des problèmes liés au manque de froid hivernal, qui soulèvent le problème du choix des variétés. L'élevage est également directement concerné, en particulier parce qu'il a le plus souffert des étés 2003 et suivants. Les questions à court terme portent sur la gestion des épisodes de sécheresse, avec la précaution première de constituer des stocks fourragers en prévision, et les horizons 2050 ou 2100, s'ils font l'objet d'études prospectives de plus en plus détaillées, n'ont pas d'intérêt stratégique immédiat. Il en va de même pour les cultures annuelles, dont on peut estimer que le temps de réponse, plutôt de l'ordre de 5 à 10 ans, n'impose pas de tenir compte en pratique d'échéances aussi lointaines. Le débat a tourné beaucoup autour de l'adaptation à la sécheresse (voir l'expertise collective de l'INRA à ce sujet, Amigues et al. 2006), et il a rebondi récemment à propos d'études, associant les instituts techniques (Arvalis) et l'INRA, qui attribuent un rôle majeur au réchauffement récent dans la stagnation des rendements observée depuis une quinzaine d'années (Gate et al 2007). La capacité d'adaptation finalement assez rapide de ces systèmes de production à base de cultures annuelles est d'ailleurs bien illustrée par deux exemples concrets d'adaptation de la part des acteurs : d'une part, l'avancée des dates de semis, observée aussi bien pour le blé ou l'orge d'hiver que pour le maïs, et qui atteint deux à trois semaines ; la réduction des surfaces irriguées en maïs, de l'ordre de 10 à $15 \%$ au niveau national (mais aussi 30 à 40\% dans certaines zones de Poitou-Charentes ou du Sud-Ouest en deux années après 2006).

\section{Conclusion}

Pour la France, dans un premier temps, un réchauffement de $1^{\prime}$ ordre de $2^{\circ} \mathrm{C}$ pourrait être favorable pour l'agriculture et la sylviculture, au moins dans le Nord et ne provoquerait qu'un léger déplacement d'équilibre, restant dans les limites des capacités d'adaptation presque traditionnelles. Celui-ci sera, pour partie, technique, avec une forte interaction entre génétique et pratiques culturales, mais devra également tenir compte du contexte économique et social. Les acteurs pourront disposer des solutions techniques qui commencent à faire l'objet des études que nous venons d'évoquer, mais bien évidemment ce sont eux qui vont détenir les clés de leur mise en pratique. Nous ne disposons pas, à l'heure actuelle, de données d'enquête sérieuses sur leur appréhension de la question de l'impact du réchauffement climatique, et il est possible que la grande attention manifestée par les milieux professionnels ne soit pas tout à fait représentative de la perception par les acteurs de terrain. Cependant, à l'occasion de nombreux contacts avec les agriculteurs, qu'ils soient céréaliers, viticulteurs ou éleveurs, et les sylviculteurs, nous avons pu constater que l'observation des impacts du réchauffement faisait l'objet d'un consensus très large, en tout cas depuis 2003. D'une attention polie en 2002, on est passé à un intérêt soutenu sur les modalités d'adaptation à la variabilité actuelle, et d'interrogations teintées d'inquiétude sur le futur proche.

Pour des horizons plus lointains, il est difficile à l'heure actuelle de cerner les conséquences d'un réchauffement de 4 à $5^{\circ} \mathrm{C}$, car il n'est 
pas acquis que les modèles utilisés fassent intervenir les informations pertinentes sur le fonctionnement des cultures dans des gammes, en particulier thermiques, se situant hors de ce que ces cultures ont expérimenté dans le passé. Mais des raisonnements plus simples, basés sur les observations de la localisation géographique des potentialités, conduisent à penser qu'un tel réchauffement provoquerait sans doute des ruptures significatives. Son impact sur les écosystèmes et les secteurs d'activité serait alors tel qu'il est illusoire de le pronostiquer dans un climat totalement bouleversé.
$R \cdot \dot{E} \cdot F \cdot \dot{E} \cdot R \cdot E \cdot N \cdot C \cdot E \cdot S$

AMIGUES J.P., DEBAEKE P., ITIER B., LEMAIRE G., SEGUIN B., TARDIEU F., THOMAS A., éditeurs., 'Sécheresse et agriculture. Adapter l'agriculture à un risque accru de manque d'eau'. Expertise scientifique collective, synthèse du rapport, INRA (France), 72 p., 2006.

BADEAU V., DUPOUEY J.L., CLUZEAU C., DRAPIER J., « Aires potentielles de répartition des essences forestières d'ici $2010 »$, Rendezvous techniques de l'ONF, hors-série $n^{\circ} 3$, "Forêts etmilieux naturels face aux changements climatiques », 2007, pp. 62-66

CLUZEAU C., DRAPIER J., VIRION R., DUPOUEY J.L., Changements à long terme de la végétation forestière : apport des données de l'Inventaire forestier national, Rapport final, Projet GIP-ECOFOR, Programme " Forêt et Modifications de l'Environnement », 2001.

DELECOLLE R., SOUSSANA J.F., LEGROS J.P., « Impacts attendus des changements climatiques sur l'agriculture française ». C.R. Acad. Agric. Fr., vol. 85,1999, pp. $45-51$.

DOMERGUE M., LEGAVE J.M., CALLEJA M., MOUTIER N., BRISSON N., SEGUIN B. "Réchauffement climatique : quels effets sur la floraison chez trois espèces fruitières ?» Arboriculture fruitière, vol. 578, 2004, pp. 27-33.

DUCHÊNE E ., SCHNEIDER C., « Grapevine and climate change: a glance at the situation in Alsace ", Agron for sust. dev., vol. 25, 2005, pp. 93-99

DUPOUEY J.L., BODIN J., « Déplacements 
déjà observes des espèces végétales: quelques cas emblématiques, mais pas de migrations massives ", Rendez-vous techniques de l'ONF, hors-série no3, "Forêts et milieux naturels face aux changements climatiques », 2007, pp. 34-39

EASTERLING W., APPS M., « Assessing the consequences of climate change for food and forest resources: a view from IPCC », Climatic change, vol.70, 2005, pp. 165-189

EASTERLING, W., AGGARWAL, P., BATIMA, P., BRANDER, K., ERDA, L., HOWDEN, M., KIRILENKO, A., MORTON, J., SOUSSANA, J. F., SCHMIDHUBER, J., TUBIELLO, F. « Food, fibre, and forest products » in Climate Change 2007: Climate Change Impacts, adaptations and vulnerability, IPCC Working Group II, Cambridge University Press, Cambridge, England, 2008.

EEA., Impacts of Europe changing climate. An indicator-based assessment, EEA report $\mathrm{n}^{\circ}$ 2/2004, 2004, 107 p.

Forêt méditerranéenne (2008). Changements climatiques en forêt méditerranéenne, tome XXIX, vol. 2., 2008, 264 p.

GANICHOT B., Évolution de la date des vendanges dans les Côtes du Rhôneméridionales. Actes des $6^{\mathrm{e}}$ Rencontres Rhodaniennes. Institut Rhodanien. Orange, France, 2002, pp. 38-41.

GATE P., BLONDOT A., GOUACHE D., " Impact du changement climatique sur la croissance du blé en F rance. Quelles solutions et quelles actions à développer », actes du colloque 'biodiversité et changements climatiques' organisé par l'AFPP, Montpellier, 21 octobre 2008.

GIEC (2007a) : « Climate change 2007: the physical science basis. Summary for policymakers ». Contribution of Working Group I to the fourth assessment report of the Intergovernmental Panel on climate change, website: www.ipcc.ch.

GIEC (2007b) : «Climate change 2007: impacts, adaptation and vulnerability ». Summary for policymakers. Contribution of Working Group II to the fourth assessment report of the Intergovernmental Panel on climate change, website: www.ipcc.ch.

HOWDEN M., SOUSSANA J.F., TUBIELLO F.N., CHTETRI N., DUNLOP M., AGGARWAL P.K., Adapting agriculture to climate change, PNAS, vol 104, 2007, pp. 19691-19696.

KLÖTZLI F., WALTHER G.R. (eds.), Conference on recent shifts in vegetation boundaries of deciduous forests, especially due to generam global warming, Birkhäuser, Basel, 1999.

LEGAY M., MORTIER F. MENGINLECREULX P., CORDONNIER T., « La gestion forestière face aux changements climatiques: tirons les premiers enseignements », Rendez-vous techniques de l'ONF, hors-série $n^{\circ} 3$, "Forêts et milieux naturels face aux changements climatiques », 2007, pp. 95-102.

LENOIR J., GÉGOUT J.C., MARQUET P.A., DE RUFFRAY P., BRISSE H., «A significant upward shift in plant species optimum elevation during the 20th century ", Science, vol 320, 2008, pp. 1768-1771

LOUSTAU D., ed., Séquestration de carbone dans les grands écosystèmes forestiers en France, rapport final du projet CARBOFOR, INRA Bordeaux-Pierroton, 2004.

RIGOLOT E., « Impact du changement climatique sur les feux de forêt ", Forêt 
méditerranéenne, tome XXIX, vol 2, 2008, pp. 167-176.

ROQUES A., NEGEILSEN L.M., " Impact du réchauffement global sur les populations d'insectes forestiers ", Rendez-vous techniques de l'ONF, hors-série $n^{\circ} 3$, "Forêts et milieux naturels face aux changements climatiques ", 2007, pp. 40-46.

SEGUIN B., GARCIA DE CORTAZAR I., «Climate warming: consequences for viticulture and the notion of terroirs in Europe ", Acta Horticulturae, vol. 689, 2005, pp. 61-71.

SEGUIN B., BRISSON N., LOUSTAU D., DUPOUEY J.L. " Impact du changement climatique sur l'agriculture et la forêt $»$, in L'Homme face au climat, actes du symposium du Collège de France, Paris, 12-13 oct 2004, ed Odile Jacob, 2006, pp. 177-204.

SOUSSANA J.F., " Changement climatique. Impacts possibles sur l'agriculture et adaptations possibles ", Demeter, Armand Colin, Paris, 2001, pp. 195-222.
$\mathrm{R} \cdot \mathrm{E} \cdot \mathrm{S} \cdot \mathrm{U} \cdot \mathrm{M} \cdot \mathrm{E}$ Le changement climatique va profondément affecter les productions agricoles et sylvicoles. Les effets en termes de potentialité de production seront très variables suivant les régions et les couvets végétaux. Ils peuvent se traduire par une tendance globale à une stimulation modérée en climat tempéré et pour un réchauffement limité à $2^{\circ}-3^{\circ} \mathrm{C}$. Au-delà, comme pour les basses latitudes, des températures excessives et la fréquence accrue des sécheresses conduiront à des effets négatifs. Ces impacts vont amener à des adaptations, qui combineront des modifications locales des systèmes de culture ou de gestion, ainsi que déplacements géographiques des systèmes de production. Les acteurs de terrain, qui ont déjà pu observer des évolutions notables en conséquence du réchauffement récent, y sont assez bien préparés

\section{Summary}

Climatic change will deeply impact agricultural and forest productions. The effects in terms of production potentialities will be largely variable between regions and productions. They may result in a global tendency to slightly increase in temperate climates as far as the warming is limited to $2^{\circ} \mathrm{C}-3^{\circ} \mathrm{C}$. Above, as for low latitudes, the overall effect will be negative due to excessive temperatures and increased drought frequencies. These impacts will induce adaptive actions. They will combine changes in cropping or forestry systems on a local scale, together with geographical shifts in production systems. Field actors have been already able to observe significant changes due to the recent warming and seem to be well prepared for this adaptation 